\title{
Analyze the driving module of spiral robot by FEA and genetic algorithm
}

\author{
Xi-guang FENG*, Yu-xia ZHAO, Jie-jian DI, and Quan-liang ZHAO \\ College of Mechanical and Materials Engineering, North China University of Technology, Beijing, \\ 100144, China
}

\begin{abstract}
The existing whip tail drive size parameter is obtained in the prototype a number of orthogonal experiments to obtain a value. In this paper, a new method is proposed. According to the resistance theory, a propulsive force calculation model is established, and then the local optimal solution is obtained through the combination of finite element method and genetic algorithm. The calculation is small and the thinking is clear. The obtained parameters are of reference to the prototype experiment.
\end{abstract}

\section{Introduction}

Swimming micro robot refers to the micro robot that moves in the liquid environment through swimming [1]. They can enter into all kinds of places which are full of liquid and has narrow space to work and explore. Spiral propulsion swimming robot is inspired by the movement of flagella bacteria in liquid environment, it has the characteristics of high efficiency and high adaptability. However, the driving system parameters of the existing spiral swimming robot are to get a better value by conducting orthogonal experiment for many times on the prototype, which takes much time and has complicated process[2].

This paper presents a new method. First establish a simple propulsive force calculation model according to the motion characteristics of bacterial flagella, and then set up bacterial flagella physical evaluation standard in COMSOL Multiphysics according to this model, then in MATLAB, taking calculation model as objective function to obtain the extreme value of the objective function according to the robot size in a selection interval of flagellum parameter, and then substitute into the above-mentioned evaluation standard in COMSOL to obtain a better solution. This method is small in workload and time saving. It can provide a better parameter value before the prototype experiment, reduce the complexity of the prototype experiment, and the data obtained are scientific enough.

\section{Resistance theory}

Resistance theory is a simple theory of propulsive force calculation. It can be applied to the flagellum model to get the force, torque and velocity of approach when interacting with the surrounding fluid [3].

\footnotetext{
*Corresponding author: fxg0828@126.com
} 
Figure 1 is a simplified flagellum tail model, and the expressions and relationships of each parameter are shown as illustrated. Other geometric relationships for model parameters are:

$$
\begin{gathered}
\theta=\arctan \left(\frac{\lambda}{2 \pi(A-a)}\right) \\
\mathrm{l}=\mathrm{n} \times \lambda
\end{gathered}
$$

In the formula, $\mathrm{n}$ is the number of spiral circle.

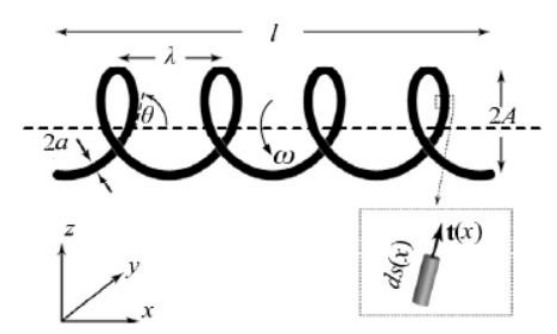

Fig. 1. Simplified model of spiral tail.

Figure 2 is the simple representation of forces exerted on the structure. When the spiral tail rotates, dividing the helical flagella into some small segments, take a length of ds, the velocity is resolved as normal velocity $V_{n}$ and tangential velocity $V_{t}$ shown in figure 1 , positive pressure $\mathrm{d} F_{n}$ and tangential resistance $\mathrm{d} F_{t}$ are applied to each small segment by the surrounding fluid[4], and the sizes respectively are:

$$
\mathrm{d} F_{n}=C_{n} V_{n} d s \quad d F_{t}=C_{t} V_{t} d s
$$

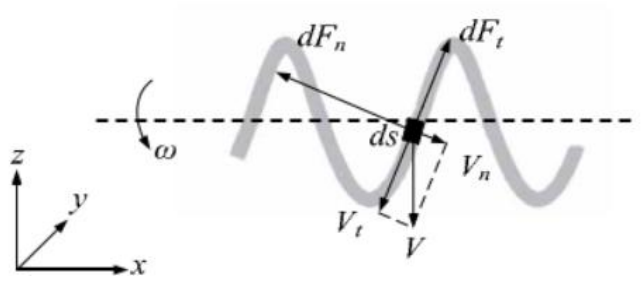

Fig. 2. Force decomposition drawing of flagellum unit.

Thereinto, $C_{t}$ and $C_{n}$ refer to the positive pressure coefficient and the tangential resistance coefficient.

The force and moment acting on the axis of the small segment is obtained:

$$
\mathrm{d} F_{x}=d F_{t} \sin \theta-d F_{n} \cos \theta \quad \mathrm{d} L_{x}=-A\left(d F_{t} \cos \theta+d F_{n} \sin \theta\right)
$$

The force and moment of the surrounding environment fluid to the whole flagellum tail are obtained by the integral:

$$
\begin{gathered}
F_{x}=\int_{0}^{l} d F_{x}=2 \pi a \int_{0}^{l}\left[\left(C_{t}-C_{n}\right) \omega A \sin \theta \cos \theta-\left(C_{t} \cos ^{2} \theta+C_{t} \sin ^{2} \theta\right) U_{x}\right] d s \\
T_{y}=\int_{0}^{l} A d F_{y}=2 \pi a \cdot A \int_{0}^{l}\left[\left(C_{n}-C_{t}\right) U_{x} \cos \theta \sin \theta+\left(C_{n} \cos ^{2} \theta+C_{t} \sin ^{2} \theta\right) \omega A\right] d s
\end{gathered}
$$

In the formula, $\mu$ is the coefficient of kinetic viscosity, and $C_{n}$ and $C_{t}$ can use the accurate expression put forward by Cox et al.

$$
C_{n}=\frac{4 \pi \mu}{\ln \left(\frac{2 \lambda}{a}\right)+0.193} \quad C_{t}=\frac{2 \pi \mu}{\ln \left(\frac{2 \lambda}{a}\right)-0.087}
$$


According to the above formula, we can know the dynamic parameters of the spiral swimming micro robot's tail propulsion force, velocity of approach and turning moment according to the known rotate speed of the spiral drive[5].

\section{Simulation modeling method}

\subsection{Three-dimensional geometrical modeling}

The model geometry is established by using the COMSOL Multiphysics rotating machinery- laminar flow module, and the model geometric parameters of the initial value setting are listed as table 1 .

Table 1. Initial values setting of the model geometric parameters.

\begin{tabular}{|l|l|l|l|l|l|}
\hline Parameter & $\begin{array}{l}\text { Minor } \\
\text { diameter/a }\end{array}$ & $\begin{array}{l}\text { Thread } \\
\text { pitch } / \lambda\end{array}$ & Radius/A & $\begin{array}{l}\text { Cylinder } \\
\text { number } / \mathrm{n}\end{array}$ & Overall length/ \\
\hline Set value & $1 \mathrm{~mm}$ & $3 \mathrm{~mm}$ & $3 \mathrm{~mm}$ & 5 & $15 \mathrm{~mm}$ \\
\hline
\end{tabular}

According to the above-mentioned computational expression and the geometric parameters of table 1 , the values of other calculating parameters can be obtained, as shown in table 2 .

Table 2. Other Calculating Parameters.

\begin{tabular}{|l|l|l|l|l|}
\hline Parameter & $\begin{array}{l}\text { Coefficient of } \\
\text { kinetic viscosity } / \mu\end{array}$ & $\begin{array}{l}\text { Positive pressure } \\
\text { coefficient } / C_{n}\end{array}$ & $\begin{array}{l}\text { Tangential resistance } \\
\text { coefficient } / C_{t}\end{array}$ & $\begin{array}{l}\text { Helix } \\
\text { angle } / \theta\end{array}$ \\
\hline $\begin{array}{l}\text { Calculate } \\
\text { d }\end{array}$ & $0.001 \mathrm{~Pa} \cdot \mathrm{s}$ & $0.0044838 \mathrm{~Pa} \cdot \mathrm{s}$ & $-0.0023293 \mathrm{~Pa} \cdot \mathrm{s}$ & $0.378 \mathrm{rad}$ \\
\hline
\end{tabular}

In consideration of the near wall effect of the spiral body rotation, a cylindrical flat bottom tank with a radius of $30 \mathrm{~mm}$ and a height of $150 \mathrm{~mm}$ is added at the same time. The flat bottom tank area removing spiral line drive is set as a fluid, and the spiral line of the rotating motion is set as a rigid body.

The constructed geometry of the completed 3D geometric model consists of "4 domains", "42 boundaries", "76 edges" and "43 vertices", as shown in figure 3.

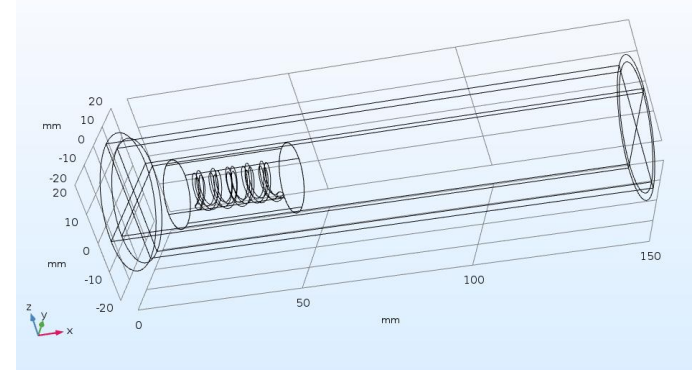

Fig. 3. Geometric model.

The spiral speed is set to $100 / \mathrm{min}$. When setting the mesh, the hexahedral mesh is generated through the swept operation with the maximum unit size $12 \mathrm{~mm}$, the minimum unit size $1.5 \mathrm{~mm}$ and the fixed unit number of 100 on spiral drive, and the rest part is free tetrahedral mesh generation. The final complete mesh consists of "82432" domain units, 13892 boundary elements, and 1108 edge units, as shown in figure 4. 


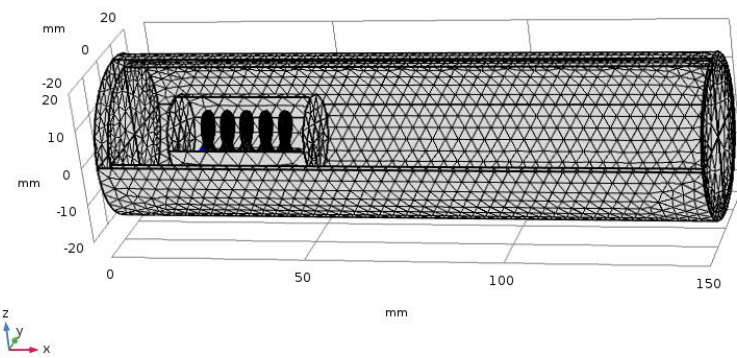

Fig. 4. Model after completion of mesh generation.

\subsection{Result analysis}

As the result is changed with time, the transient research is done on the setting of solver. In the configuration of the solver, the step size is $0.25 \mathrm{~s}$, and a total of $20 \mathrm{~s}$ computing setting is set.

According to the foregoing discussion, the spiral line is integrated and the force and moment of the spiral line in motion is calculated respectively. The results obtained are shown in figure 5 .

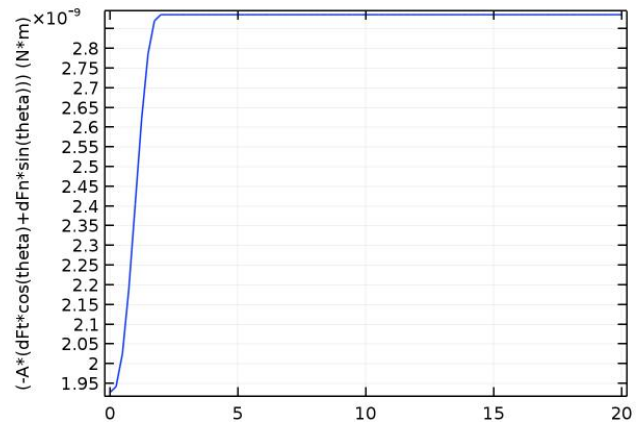

Fig. 5. X Direction force and moment of spiral line at 0-20s.

According to the chart, as the step function acts on the spiral speed, when the spiral drive is gradually balanced in the fluid, the force decreases to the equilibrium point, and the moment gradually increases to the equilibrium point.

\section{Optimal solution of genetic algorithm}

Genetic algorithm has the advantages of simplicity and strong robustness[6]. In this case, by finding the extreme value of the velocity formula, the independent variable which makes the objective function get the maximum value is obtained, and the better parameter value of the model is obtained.

\subsection{Variables and the objective function}

According to the robot size, set the minor diameter a to be $1 \mathrm{~mm}$, other size parameters and calculating parameters, such as the helix angle $\theta$, overall length 1 , tangential resistance coefficient $C_{n}$ and positive pressure coefficient $C_{t}$, will be affected by the change of spiral tail drive section radius $A$ and thread pitch $\lambda$, so choose $A$ and $\lambda$ as design variables. 
According to the above-mentioned formula of the resistance theory, When the flagellum travels in the liquid environment, it is in the equilibrium state when the above force and is zero, thus the formula of velocity of movement for the flagellum is as follows:

$$
\mathrm{U}_{\mathrm{x}}=\frac{\sin \theta \cos \theta\left(\mathrm{C}_{\mathrm{t}}-\mathrm{C}_{\mathrm{n}}\right)}{\left(\mathrm{C}_{\mathrm{n}} \sin { }^{2} \theta+\mathrm{C}_{\mathrm{t}} \cos { }^{2} \theta\right)} \omega \mathrm{A}
$$

As the objective function of the genetic algorithm, each size and the calculation parameters in the formula have been mentioned above.

\subsection{Process and result analysis}

The maximum iteration number of the set population is 50 times. The value range of $\mathrm{A}$ is $3 \sim 8 \mathrm{~mm}$, and the value range of $\lambda$ is $2 \sim 10 \mathrm{~mm}$. Through a series of operations, such as selection, hybridization, mutation, etc, and multiple program execution, the change curve of the final objective function is shown in figure 6 .

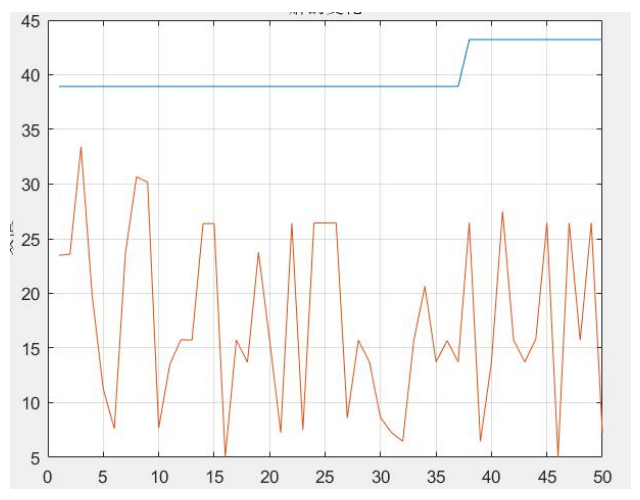

Fig. 6. Change curve of the objective function.

The locally optimal solution selected by the design variables is $A=3.0010 、 \lambda=$ 3.9987 , so the values of two selected are $3 \mathrm{~mm}$ and $4 \mathrm{~mm}$ respectively. The locally optimal solution selected by genetic algorithm is ideal on this problem of finding solution in this paper, but because the genetic algorithm has the disadvantages of premature convergence, low searching efficiency in later period, so that the final search results is the locally optimal solution rather than the globally optimal solution.

\section{Contrastive analysis}

After the operation of solving the extreme value by genetic algorithm, the locally optimal solution is substituted into COMSOL Multiphysics to establish a new 3D geometric model, and the other geometric and calculating parameters are listed in table 3.

Table 3. Geometric and calculating parameters derived from locally optimal parameters.

\begin{tabular}{|c|c|c|c|c|}
\hline Parameter & $\begin{array}{c}\text { Overall } \\
\text { length/ }\end{array}$ & $\begin{array}{c}\text { Positive pressure } \\
\text { coefficient } / C_{n}\end{array}$ & $\begin{array}{c}\text { Tangential resistance } \\
\text { coefficient } / C_{t}\end{array}$ & Helix angle/ $\theta$ \\
\hline Calculated & $30 \mathrm{~mm}$ & $0.00421 \mathrm{~Pa} \cdot \mathrm{s}$ & $-0.0024982 \mathrm{~Pa} \cdot \mathrm{s}$ & $0.2091 \mathrm{rad}$ \\
\hline
\end{tabular}

The spiral tail driving model of the new parameters is calculated, and the other mesh and component settings are unchanged, and the results are shown in figure 7. 


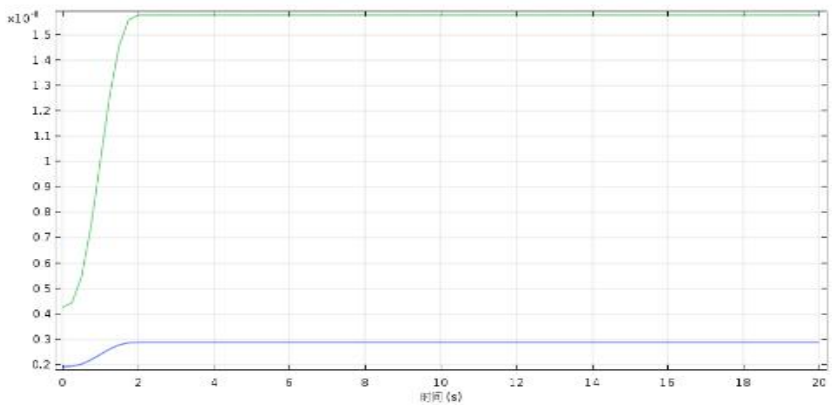

Fig. 7. X Direction force and moment of spiral tail motion after optimization at 0 20s.

Comparing the two curves in the figures, the force and moment results of the new spiral driving parameters and initial value parameters in the $\mathrm{X}$ direction have greatly improved, according to the resistance theory, the spiral driving of new parameters will get faster velocity of approach than before, therefore, analyzing this case by the finite element method combined with genetic algorithm is feasible.

\section{Conclusion}

The driving system parameters of the existing spiral swimming robot are to get a better value by conducting orthogonal experiment for many times on the robot prototype, which is not scientific enough, and the expression is also vague. This paper presents a new method. First establish a simple propulsive force calculation model according to the motion characteristics of bacterial flagella, and then set up bacterial flagella physical evaluation standard in COMSOL Multiphysics according to this model, then in MATLAB, taking calculation model as objective function to obtain the extreme value of the objective function according to the robot size in a selection interval of flagellar parameter, and then substitute into the above-mentioned evaluation standard in COMSOL to obtain the locally optimal solution. This method reduces the experimental workload, and the data has the advantages of intuitive and strong reference, and the desired results are obtained through this case.

\section{References}

1. Li Yajuan. Research on Spiral Propulsion Biomimetic Swimming Robot [D], Nanjing University of Aeronautics and Astronautics, 2013

2. Zhang Linyan, Zhang Yongshun, Xu Liang. The development and Prospect of medical micro robot in the body [J]. machinery manufacturing, 2006 (10): 33-37.

3. Zhu Qianyun. Research on the Motion Characteristics of Micro-robot Simulating Flagellum Swimming [D], Nanjing University of Aeronautics and Astronautics, 2015

4. Robot E M A. Design of Micro Robot for Minimally Invasive Surgery[J]. International Journal of Robotics and Automation (IJRA), 2013, 2(1): 35-44.

5. Song Xiaofeng. Talking about the development and research status of micro robot [J]. hydraulic and machine tools, 2004 (8): 1-2149.

6. Xie Chengwang, Hu Jiabao. Extreme Value Problem Solution Based on Genetic Algorithm [J], Journal of Wuhan Institute of Science and Technology, 2004 (02): 64-67. 